\section{Nachhaltige Entwicklung: Vom Schlagwort zur politischen Strategie}

\author{
Berlin, 28. September 2001
}

\section{Bericht zur Auftaktveranstaltung des Rates für Nachhaltige Entwicklung, zusammenge- stellt durch die Geschäftsstelle des Rates}

Am 28. September d.J. fand in Berlin die Auftaktveranstaltung des Rates für Nachhaltige Entwicklung $^{1}$ statt. Sie stand unter dem Motto „Nachhaltige Entwicklung: Vom Schlagwort zur politischen Strategie“.

In seiner Begrüßung hob Prof. Dr. Josef Sayer besonders den internationalen Kontext nach den Anschlägen auf das World Trade Centre und das Pentagon am 11. September 2001 hervor, vor dessen Hintergrund die Arbeit des Rates jetzt auch zu sehen sei. Der internationale Aspekt sei bei der Entwicklung einer nationalen Nachhaltigkeitsstrategie von großer Bedeutung.

\section{Nachhaltige Entwicklung - Herausforderung für die Politik (Dr. Volker Hauff, Vorsitzender des Rates für Nachhaltige Entwicklung)}

In der Eröffnungsrede ging Dr. Volker Hauff auf die Erwartungen ein, die dem Rat entgegengebracht werden, und stellte den Bezug zur aktuellen weltpolitischen Lage her. Von entscheidender Bedeutung sei für den Rat dabei die Festschreibung eindeutiger Ziele: „Eine unserer ersten Empfehlungen an die Bundesregierung ist es, quantifizierte Ziele zum Eckpunkt der nationalen Nachhaltigkeitsstrategie zu machen“. Bereits bei der Definition des nationalen Klimaziels habe sich dieses Vorgehen bewährt. Mit klaren Zielgrößen habe Deutschland hier einen wesentlichen Beitrag geleistet. Mit seiner Arbeit wolle der Rat dazu beitragen, dass die Bundesregierung zu solchen konkreten Zielen in drei Schwerpunktbereichen - der Landwirtschafts-, Energie- und Verkehrspolitik - kommt. Bereits seit Mitte des Jahres habe der Rat dazu drei Arbeitsgruppen zu den Themenbereichen „Energie und Klimaschutz“, „Mobilität und Verkehr“ sowie „Landwirtschaft, Ernährung, Gesundheit und
Umwelt" berufen, deren Ansätze auf der Auftaktveranstaltung diskutiert wurden.

Der ,weitgehend ungebremste Flächenverbrauch" in der Bundesrepublik sowie die „Bekämpfung der Armut, des Hungers und der Ressourcenschutz" seien für den Rat zwei weitere Themenbereiche, bei denen ein konkreter Beitrag Deutschlands notwendig sei. Hier habe sich der Rat ebenfalls die Definition klarer Ziele in seine Agenda geschrieben.

Schließlich erläuterte der Vorsitzende den Fahrplan des Rates für die nächsten Monate:

- Es soll ein breiter und öffentlicher Meinungsaustausch stattfinden.

- Ende November wird ein Projektforum zur Nachhaltigen Entwicklung eingeric htet.

- Der Rat wird der Bundesregierung empfehlen, weitere Pilotprojekte zur Nachhaltigen Entwicklung aufzugreifen.

- Im November soll ein öffentliches Gespräch zu Zielen der Nachhaltigen Entwicklung beginnen. ${ }^{2}$

\section{Die politischen Ziele der nationalen Nach- haltigkeitsstrategie der Bundesregierung und der Weg bis Johannesburg 2002 (Staatsminister Hans Martin Bury)}

Hans Martin Bury, Staatsminister im Bundeskanzleramt und Vorsitzender des Staatssekretärsausschusses der Bundesregierung zur Nachhaltigen Entwicklung (,Green Cabinet"), stellte im Anschluss die Überlegungen der Bundesregierung zur Nachhaltigen Entwicklung sowie die bisherigen Arbeitsergebnisse des Staatssekretärsausschusses vor.

Bury erläuterte für die Bundesregierung die „Koordinaten der Nachhaltigkeit“":

- Generationengerechtigkeit (z. B. Sanierung der Staatsfinanzen, Rentenreform, ,ein Quantensprung bei der Energie- und Ressourceneffizienz", Umwelt),

- Lebensqualität (z. B. eine neue Agrarpolitik, Umwelt, Schulen, Bildung, Arbeitsplätze),

- Sozialer Zusammenhalt (z. B. die Vermeidung einer Spaltung der Gesellschaft in Gewinner und Verlierer),

- Internationale Verantwortung (z. B. Intensivierung der Entwicklungszusammenarbeit, Marktöffnung für Entwicklungslän- 
der, Aktionsprogramm Armutsbekämpfung der Bundesregierung).

Die Strategie soll einem dreifachen Ansatz (Leitbild, prioritäre Handlungsfelder und konkrete Pilotprojekte) folgen und thematisch breit angelegt werden. Es sei beabsichtigt, die Strategie prozessorientiert und im Dialog zu erarbeiten.

Ein „Managementkonzept der Nachhaltigkeit" soll auf zwei Pfeilern aufgebaut sein: Zum einen sollen die (bislang nur umweltbezogenen) Managementregeln erweitert werden, zum anderen sollen Indikatoren (insb. Schlüsselindikatoren) und (qualitative und quantitative) Ziele formuliert werden, um eine Bilanz der Nachhaltigen Entwicklung in Deutschland zu ermöglichen. So weit wie möglich sind quantifizierte Ziele vorgesehen.

$\mathrm{Zu}$ den Handlungsschwerpunkten Energie, Mobilität und Landwirtschaft sollen konkrete Maßnahmen benannt werden. Weitere, jedoch nicht in gleichem Maße zu vertiefende Handlungsfelder seien beispielsweise die demografische Entwicklung und der Bereich Hochschule und Bildung. Es soll deutlich werden, dass die Nachhaltigkeitspolitik ein zeitlich weit gestrecktes Politikfeld sei.

Die Aufgabe „Nachhaltigkeit" gehe über die Vorlage einer Nachhaltigkeitsstrategie in Johannesburg hinaus: Sie bestimme maßgeblich die Reformpolitik der Bundesregierung.

Bury erläuterte zudem die Pilotprojekte des Staatssekretärsausschusses. Um den innovativen Charakter dieser Projekte hervorzuheben, betonte er beispielhaft, dass das erste ZeroEmission-Car in Deutschland vom Band gehen solle.

\section{Diskussion}

Im Anschluss an die Reden von Herrn Dr. Hauff und Herrn Staatsminister Bury gab es Gelegenheit zur Diskussion.

Nicht auf alle Fragen und Kommentare konnten die Angesprochenen erschöpfend eingehen. Die Äußerungen der Vortragenden zu den Redebeiträgen werden im Folgenden kurz zusammengefasst.

Bury betonte, dass im Bereich der Entwicklungspolitik privaten Beiträgen ein größeres Gewicht zukommen müsse als bislang. Zur
Frage der Partizipation sowie zur Zusammenarbeit zwischen Rat und Staatssekretärsausschuss wies er darauf hin, dass die erste gemeinsame Sitzung von Rat und Staatssekretärsausschuss bewusst erst kurz nach dieser Auftaktveranstaltung stattfände. Eine Verzahnung zwischen den beiden Gremien sei durch intensiven Austausch gesichert. Zur aus dem Publikum angesprochenen Beteiligung der Länder an der Erarbeitung der Nationalen Nachhaltigkeitsstrategie lud Bury die Länder ein, schon heute die konkreten Kooperationsangebote der Bundesregierung, zum Beispiel zur Kofinanzierung einer modifizierten Agrarpolitik, aufzugreifen.

Auf zukünftige Arbeitsschwerpunkte des Rates angesprochen, bemerkte Hauff, dass der Rat sich nach seiner Meinung angesichts der eher oberflächlichen öffentlichen Diskussion um die Ökosteuer auch zu derartigen umstrittenen Themen äußern könnte.

Er wies darauf hin, dass es kein allgemein gültiges Nachhaltigkeits-Modell geben könne es gehe darum, zu diskutieren, was genau Nachhaltigkeit im Einzelfall sei. Für die Nachhaltigkeitsdefinition wünscht er sich ein wenig mehr Schärfe: Bislang sei das Verständnis, dass Nachhaltigkeit alles sei, was gut und schön ist, nicht nachhaltig sei alles, was schlecht sei. Der Charakter der Diskussion sei stellenweise zu moralisierend.

Zur Frage nach der - aus dem Publikum angesprochenen „mangelnden“ - Vertretung der jungen Generation im Rat, führte Hauff aus, dass der Rat beabsichtigt, in seinen Arbeitsgruppen auch Vertreter weiterer Gruppen, und insbesondere von Jugendorganisationen, von Fall zu Fall an den Arbeiten zu beteiligen und im übrigen für Anregungen aller sozialer Gruppen offen sei.

\section{Kommunikation: Wie den Gedanken der Nachhaltigkeit vermitteln?}

Vor der Mittagspause übernahm der Geschäftsführer des Rates, Herr Dr. Bachmann, die Mbderation zur Frage: Wie kann der Gedanke der Nachhaltigkeit vermittelt werden? Kommunikation zur Nachhaltigkeit solle dazu befähigen, richtige Fragen zu stellen und Quer-Denken zulassen. Zu Recht sei bereits auf die kulturpolitische Dimension der Nachhaltigkeit hinge- 
wiesen worden: Bildung für eine nachhaltige Entwicklung habe der Bund-Länder-Bildungskongress im Juni dieses Jahres angesprochen.

Kommunikation gehe nicht nur über den Kopf. Wer Verhalten ändern wolle, müsse auch die Sinne ansprechen. Ein Beispiel, das zugleich auch ein Experiment bei dieser Art von Veranstaltung sei, sei das Musikstück, Anything at all", das vom Trio Inner Life extra für diese Veranstaltung komponiert wurde. Das Trio wiederholte das Stiick, Anything at all" in den Pausen und am Ende der Veranstaltung, wobei es das Stück gemäß der Stimmung der Teilnehmerinnen und Teilnehmer mit Tempo, $\mathbf{h}$ strumenteneinsatz und Lautstärke variierte und damit der Veranstaltung unmittelbar ein kommunikatives Feedback gab. Anschließend wurde unter dem Motto „Verlassen Sie diesen Raum so, wie Sie ihn vorfinden möchten" ein Kurzfilm vorgestellt, der den Gedanken der Nachhaltigkeit im Spiegel von Meinungen und Haltungen in der Bevölkerung darstellte und den Rat und seine Mitglieder sowie die Entwicklung erster Medien (u. a. Internet, Video) des Rates vorstellte.

\section{Die Arbeitsforen}

Am Nachmittag der Auftaktveranstaltung wurden in drei Arbeitsforen die konkreten Ergebnisse der bisherigen Ratsarbeit kurz vorgestellt und mit den Teilnehmern diskutiert.

\section{Forum Energie und Klimaschutz}

Von Seiten des Rates bzw. der Arbeitsgruppe „Energie und Klimaschutz" nahmen Herr Grohe, Herr Prof. Dr. Jochem und Herr Weinzierl teil. Jochem führte mit einem kurzen Referat in die Diskussion ein und stellte Basisdaten und Arbeitsschwerpunkte vor. Man gehe davon aus, dass das gegenwärtige Niveau des Primärenergieverbrauchs in den Industriestaaten wesentlich gesenkt und weiter vom Wirtschaftswachstum abgekoppelt werden müsse. Wichtige Aspekte sind dabei die (gegenwärtig bereits erhöhte) $\mathrm{CO}_{2}$-Konzentration in der Erdatmosphäre und die Abhängigkeit von Lieferungen von Primärenergieträgern aus politisch instabilen Regionen. Durch Bevölkerungszuwachs und Wirtschaftswachstum werde der Ressourcenverbrauch in diesem Jahrhundert auf ein für die Erde unverträgliches Niveau ansteigen. Vor diesem Hintergrund hat die Arbeitsgruppe drei Handlungsschwerpunkte definiert:

- Begrenzung von Versorgungsrisiken

- Kostengünstige Energiedienstleistungen

- Stabilisierung der $\mathrm{CO}_{2}$-Konzentration auf einem klimaverträglichen Niveau.

Die Diskussionsteilnehmer äußerten verschiedene Erwartungen, die sich teilweise nicht nur unmittelbar an die Arbeitsgruppe richteten, sondern auch an den gesamten Rat. Die Diskussion berührte insgesamt sehr unterschiedliche Aspekte. Ein sehr zentraler Punkt war die Forderung mehrerer Teilnehmer nach quantifizierten Zielen. Solche Ziele seien vom Rat bzw. der Arbeitsgruppe aufzustellen, zumindest aber solle der Rat von der Regierung Entscheidungen darüber einzufordern. Ein weiteres herausragendes Thema war die Kommunikation. Klimaschutz- oder Nachhaltigkeitsziele generell sollten möglichst einfach vermittelbar gehalten und vom Rat intensiv kommuniziert werden. Eine vordringliche Aufgabe des Rates wurde darin gesehen, seine Anliegen und seine Ziele in breite Bevölkerungsschichten zu tragen. Weitere öffentliche Veranstaltungen des Rates sollten folgen.

Als ergänzende Leitbilder und Ziele für die Ratsarbeit wurden zusammengefasst folgende Punkte genannt:

- Der Rat solle intensiv kommunizieren, Dialog führen und auch motivieren.

- Soziale Aspekte sollten bei allen Forderungen mit berücksichtigt werden.

- Der Rat solle die globale Dimension der Nachhaltigkeit ansprechen und Kooperation auf globaler Ebene suchen.

- Bei allen Überlegungen sei das Thema Geschlechtergerechtigkeit einzubeziehen.

- Generationengerechtigkeit sei gerade bei der Energieproblematik relevant, weil Ursachen und Wirkungen von Handlungen zeitlich und räumlich oft auseinander fallen.

- Beim Vorschlag weiterer Projekte sei die Verknüpfung der drei Dimensionen der Nachhaltigkeit deutlich herauszustellen.

Die Arbeitsgruppe wurde aufgefordert, sich nicht zu technokratisch dem Thema Energie und Klimaschutz zu nähern. Besonders wichtig 
sei es, die Menschen dort abzuholen, wo sie gegenwärtig stehen.

Bei der Frage, wo der Rat bzw. die Arbeitsgruppe weitere Schwerpunkte setzten sollte, ergaben sich zusammengefasst folgende Erwartungen:

- Vor dem Hintergrund der aktuellen politischen Situation wurde das Gewicht des Aspektes der Versorgungssicherheit bestätigt.

- Eine Aussage zur Ökosteuer wird nicht kurzfristig, jedoch im Laufe des nächsten Jahres erwartet; das Thema rücke dann wieder im Zuge des Wahlkampfes in den Brennpunkt.

- Konkret soll der Rat sich für eine Kennzeichnungspflicht der Energieeffizienz von Geräten einsetzen.

- Der Rat solle sich für Entwicklung und Verbreitung schadstoffarmer Fahrzeuge einsetzen.

- Den großen Bedarf an Erneuerung von Energiesystemen in den nächsten Jahren solle der Rat aufgreifen und die Relevanz heutiger Investitionsentscheidungen für den Energieverbrauch der nächsten Jahrzehnte herausstellen.

- Die Arbeitsgruppe solle die Diskussion um die Senkenproblematik aufnehmen.

- Eine Diskussion um Lebensstile sei aufzugreifen bzw. anzustoßen. Lebensstile prägen nicht nur hier den Energiekonsum, sondern auch im Rest der Welt Deutschland sei ein großer Exporteur auch von Lebensstilen.

- Internationale Aspekte sollen eingebunden und bedacht werden.

Herr Grohe, der die Diskussion leitete, führte aus, dass der Rat bzw. die Arbeitsgruppe zwar noch keine umfassenden abschließenden Statements abgeben könne, die Diskussion habe aber gezeigt, dass die Wahl der Schwerpunktthemen den Erwartungen grundsätzlich entspreche. Insbesondere verstärkte Kommunikationsarbeit sei bereits - auch für die Arbeitsgruppe selbst - in Planung. Die von den Diskussionsteilnehmern betonten Aspekte sollen in den nächsten Sitzungen in die Arbeit der AG eingeflochten werden.

\section{Forum Mobilität und Verkehr}

Nach einem einführenden Referat von Herrn Flasbarth, der über den Stand der Arbeit der Arbeitsgruppe Mobilität und Verkehr berichtete, moderierte Hermann-Josef Tenhagen (Finanztest) die Diskussion. Im Folgenden wird die Diskussion in groben Zügen nachgezeic hnet.

Zunächst wurden die grundlegenden Vorstellungen der Teilnehmer und Teilnehmerinnen über mögliche Leitbilder und Ziele der Ratsarbeit in Sachen Mobilität zusammen gtragen. Die Diskussion war zu weiten Teilen davon geprägt, dass die Teilnehmer unterschiedliche „Brillen“ tragen, durch die sie den Bereich Mobilität betrachten. Zudem war es erforderlich, zunächst einmal die Begrifflichkeiten zu klären und Mobilität von Verkehr abzugrenzen. Einige Teilnehmer forderten eine Modernisierung der Ziele zur Mobilität: Die genannten Ziele bzw. Handlungsfelder würden zu sehr alte, im engeren Sinne verkehrsmittelorientierte, Ansätze verfolgen (z. B. „modal split") und die neueren Diskussionen nicht genügend aufgreifen, die sich von einer verkehrsmittelorientierten Herangehensweise abwenden und statt dessen auf die Frage der Befriedigung individueller Mobilitätsbedürfnisse ausgerichtet sind. Somit drängten die Teilnehmer weg von einer Analyse, die sich an Verkehrsträgern orientiert, hin zu einer an Mobilitätsanforderungen orientierten Analyse: Statt zu fragen, wie konstruiere ich z. B. ein Sprit sparenderes Auto, regten sie die Frage an, wie komme ich effizient von A nach B.

Des Weiteren wurde die Bedeutung einer Quantifizierung von Zielen sowie die Notwendigkeit der Herstellung eines Konsenses über Grundfragen betont, den es immer noch nicht gebe, obwohl bereits sehr lange über das Thema Mobilität geredet werde. Ökologische als auch soziale Ziele würden zu kurz kommen und sollten stärker betont werden. Einige Teilnehmer beschäftigte die Frage, ob die Qualität der wissenschaftlichen Erkenntnisse im Bereich Mobilität ausreichend ist. Selbst solarer Wasserstoff für Autos brächte wegen des zuvor notwendigen Aufbaus immenser Infrastrukturen erhebliche Emissionen an $\mathrm{CO}_{2}$ mit sich.

Als ergänzende Leitbilder und Ziele für die Ratsarbeit wurden folgende Punkte genannt: 
- Kostenwahrheit,

- $\quad$ eine Ent-Emotionalisierung der Mobilitätsdiskussion (durch eine Thematisierung des Zusammenhangs von Verkehr und Emotion), gleichzeitig aber auch das Ergründen der emotionalen Entscheidungsgründe der Menschen für bzw. gegen die verschiedenen Verkehrssysteme,

- neben dem „Recht auf Mobilität“ eine angemessene Abwägung anderer Rechte wie z. B. Gesundheit, Lärmschutz, Recht auf körperliche Unversehrtheit,

- Berücksichtigung des Fußgängerverkehrs,

- das Attraktiv-machen von Alternativen zum Auto.

Als wünschenswertes Handlungsfeld für die weitere Ratsarbeit im Bereich Mobilität fand ein Thema besondere Beachtung:

- Intermodalität und Verkehrsdienstleistung: Die Mobilitätspolitik sollte Rahmenbedingungen schaffen, die die Bereitstellung von Verkehrsdienstleistungen begünstigt. Analog zu einer Energiedienstleistung, bei der nicht mehr Strom verkauft wird, sondern ein warmer Raum, steht bei einer Verkehrsdienstleistung die Frage im Mittelpunkt: Wie komme ich am bequemsten (schnellsten...) zur Arbeit? Es geht um die Schaffung umweltfreundlicher/nachhaltiger Mobilitätsketten bzw. Transportketten.

Des weiteren wurden folgende Themen genannt:

- Die Ausrichtung der Siedlungsstruktur in Richtung einer nachhaltigen Mobilitätspolitik,

- Abstimmung der verschiedenen Elemente einer Mobilitätspolitik aufeinander (eine solche Abstimmung werde oft nicht mit bedacht),

- Verkehr und Konsum,

- Thematisierung von Lebensstilen,

- Verkehrs- bzw. Mobilitätserziehung, um früh ein Bewusstsein für die Problematik von Mobilität zu schaffen,

- die Berücksichtigung internationaler und intergenerationeller Aspekte,

- eine Orientierung der Politik an kurzen Wegen: auch beim ÖPNV sollte das vorrangige Ziel die Verkehrsvermeidung sein,
- die Schaffung neuer Angebote für die „Zwangsmobilität“ (im Gegensatz zum Freizeitverkehr), insbesondere arbeitsbedingte Verkehre unter die Lupe nehmen (der DBG will sich dem Thema „Arbeitswege" im Bereich der betrieblichen Bildung widmen),

- Güterverkehr (Vernetzung der Verkehrsträger).

Für Projektvorschläge seitens der Teilnehmer blieb leider nicht mehr viel Zeit. Als Stichworte wurden genannt:

- die Schaffung einer Nachhaltigkeitsregion,

- die Bildung/Förderung eines ,intermodalen Mobilitätsproviders",

- alle großen Verkehrsanbieter bieten alle Fortbewegungsarten an (,die Lufthansa fährt Fahrrad"),

- die Erstellung eines nachhaltigen Bundesverkehrswegeplans (als Gegenstück zum traditionellen Bundesverkehrswegeplan),

- die Bundesregierung solle durch ihr Handeln im Bereich Mobilität selbst als Vorbild und Modell dienen,

- innovative Mobilitätsdienstleistungen mit sauberem Antrieb.

Als Oberziel wurde das ,Zero emission vehicle“ gefordert.

Für den Rat hatten Heinz Putzhammer und Roland Heinisch die Zusammenfassung der Diskussion übernommen. Er betonte, dass die Veranstaltung zeige, dass der Rat bewusst den Kontakt mit den Engagierten im Bereich der Nachhaltigen Entwicklung sucht und halten will. Für das Ratsmitglied Heinisch stand als Erkenntnis aus der Diskussion im Vordergrund, die tatsächliche $\mathrm{CO}_{2}$-Bilanz der Brennstoffzelle kritisch zu prüfen. Ein weiteres Ergebnis der Diskussion war für ihn, dass für den Menschen als potenziellen Nutzer/Entscheider der verschiedenen Verkehrsmittel - gerade im Personenverkehr - neben den nüchternen Fakten bei der Erarbeitung von Zielen, Strategien und Maßnahmen auch viele emotionale und schwer greifbare Kriterien maßgebend seien, die man berücksichtigen müsse, wenn ein Wechsel zu nachhaltigen Formen stattfinden solle. 
Forum Landwirtschaft, Umwelt, Ernährung und Gesundheit

Frau Prof. Dr. Müller führte in das Forum ein und stellte die Mitglieder der Arbeitsgruppe Landwirtschaft, Umwelt, Ernährung und Gesundheit des Rats vor (Prof. Dr. Edda Müller, Verbraucherzentrale Bundesverband; Philip Freiherr von dem Bussche, Deutsche Landwirtschaftsgesellschaft; Prof. Dr. Sayer, Misereor, Dr. Angelika Zahrnt, BUND). Einleitend stellte Frau Müller mit einigen Folien die Arbeitsweise, das zugrunde liegende Leitbild und erste auftauchende Zielkonflikte der Arbeitsgruppe dar.

Die Arbeit orientiert sich an vier Handlungsschwerpunkten. Diese betreffen Maßnahmen, die sowohl auf kleinräumiger (regionaler) wie auch auf großräumiger (nationaler und nternationaler) Ebene Ansatzmöglichkeiten zeigen. Die Handlungsschwerpunkte betreffen:

1. die Art der Landbewirtschaftung,

2. die Art der Nachfrage nach Lebensmitteln, damit verbunden die Art der Tie rhaltung,

3. die Flächennutzung,

4. die Austauschbeziehungen von Gütern und Lebensmitteln zwischen Nord und Süd.

Die vorgeschlagenen Maßnahmen betreffen zunächst die Art der landwirtschaftlichen Produktion selbst. Die Versorgung mit Lebensmitteln, Futtermitteln und Rohstoffen soll durch die Landwirtschaft unter Berücksichtigung der Aspekte des Umwelt-, Landschafts-, Natur-, Tier- und Verbraucherschutzes erfolgen. Eng verknüpft mit der Art der Produktion ist die Nachfrage nach den Produkten aus einer solchen Produktionsweise. Dazu müssen (Preis-)Barrieren bei den Verbrauchern abgebaut werden. Ebenso sind die tiergerechte Züchtung, Tierhaltung, Fütterung sowie der tiergerechte, stressfreie Transport und die Schlachtung der Tiere als Ganzes noch nicht als Kriterien in einen kontrollierbaren Siegelstandard eingeflossen. Eine Honorierung tiergerechter Haltung erfolge noch nicht in dem Maße, wie es wünschenswert wäre.

Damit die Landwirtschaft neben ihren bisherigen Aufgaben der Lebensmittel- und Rohstofflieferung auch ihre „,neuen Aufgaben“ (z. B. Tourismus, Nachwachsende Rohstoffe, Wiederherstellung und Pflege der Kulturland- schaften) erfüllen könne, müsse sie ihre Nutzungsinteressen wirkungsvoll vorbringen können. Aus diesem Grund sei der Flächenanteil der land- und forstwirtschaftlichen Flächen auf dem heutigen Stand zu sichern. Als Konsequenz daraus müsse der Flächenverbrauch von Siedlungs- und Verkehrsflächen minimiert werden.

Die Arbeitsgruppe beschäftigt sich zudem mit dem Thema der Verteilungsgerechtigkeit, der ,global justice“. Die landwirtschaftliche Produktion in Deutschland bzw. Europa soll so betrieben werden, dass die Länder des Südens daraus keinen Schaden tragen. Durch geeignete Instrumente sollen Anreize für die Eigenproduktion von Lebensmitteln in Entwicklungsländern geschaffen werden.

Die Arbeitsgruppe Landwirtschaft hat sich über eine Leitbild, eine Vision einer nachhaltigen Landwirtschaft verständigt. Grob skizziert strebt sie als Vision eine nachhaltige Landwirtschaft an, die im Einklang mit ökologischen, ökonomischen und sozialen Kriterien erfolgt und die intergenerational und global gerecht ist. Auch in Zukunft soll es in Deutschland eine unternehmerisch handelnde Landwirtschaft geben, die maßgeblich von bäuerlichen Familien getragen wird.

Die Diskussion von Zielkonflikten nimmt in der Arbeitsgruppe einen wichtigen Raum ein. Beispiele hie rfür sind:

- Regionalisierung versus globale Gerechtigkeit: Liberalisierung des Außenhandels bei gleichzeitiger Sicherung möglichst hoher ökologischer, sozialer, Tierschutz- und Verbraucherschutzstandards. Handelt es sich um einen ökologischen, sozialen Protektionismus? Wie kann eine globale Gerechtigkeit zwischen Nord und Süd erreicht werden?

- Flächenbedarf für multifunktionale Landwirtschaft und Siedlung und Verkehr: Begrenzung des Flächenverbrauchs für Siedlungs- und Verkehrsflächen und gleichzeitig Priorität für Landwirtschaft sowie für Natur- und Artenschutz. Wie kann ein fairer Ausgleich zwischen Anforderungen seitens der Landwirtschaft und des Naturund Artenschutzes erfolgen?

- Qualitäts-Lebensmittel (Produkt- und Prozessqualität): Gesunde Lebensmittel zu erschwinglichen Preisen bei gleichzeitiger 


\begin{abstract}
Absicherung der Einkommen der Lebensmittelerzeuger und -verarbeiter. „Qualität hat ihren Preis“: Unterstützen die Verbraucher/Innen die Agrarwende durch ihr Verhalten am Ladentisch?
\end{abstract}

Herr Prof. Sayer übernahm die Moderation der Diskussion. Im ersten Diskussionsteil wurden die Erwartungen der Forumsteilnehmer an den Rat gesammelt. Im zweiten Teil der Diskussion wurden die Äußerungen zu dem grob skizzierten Leitbild der Arbeitsgruppe, den Handlungsfeldern und Zielen vom Rat aufgenommen. Den Erwartungen des Forums gemäß soll der Rat u. a.:

- einen gesellschaftlichen Dialog führen

- $\quad$ einen Beitrag zur Konfliktlösung leisten

- wissenschaftliche Erkenntnisse einbeziehen, Grenzen aufzeigen

- integrative Konzepte verfolgen, interdisziplinär arbeiten.

$\mathrm{Zu}$ dem grob skizzierten Leitbild und den Zielen der Arbeitsgruppe wurden folgende Aspekte diskutiert:

Es wird angeregt, zwischen den verschiedenen Leitbildebenen deutlich zu unterscheiden (Leitbilder für die Landwirte, die Verbraucher, den Rat für nachhaltige Entwicklung). Leitbilder sollten integrativ entwickelt werden. Die Frage wird aufgeworfen, wie das überkommene Modell der bäuerlichen Landwirtschaft weiter entwickelt werden kann und welche weiteren Modelle sinnvoll sind, um die soziale Dimension zu berücksichtigen. Der Konflikt zwischen einer ,unternehmerischen“ und einer „geförderten“ Landwirtschaft (auch im Hinblick auf das als „WTO-Modell“ angestrebte Modell der Landwirtschaft) solle gelöst werden und im Leitbild deutlich werden. Das Leitbild solle dem Prinzip der globalen Gerechtigkeit (global justice) folgen und insbesondere die Interessen der kleinen Bauern in Nord und Süd berücksichtigen. Ziele sollten nicht $\mathrm{zu}$ hoch gesteckt und die Machbarkeit der Umsetzung nicht aus den Augen verloren werden. Es werden verschiedene Konzepte angeregt, die die Zielfindung ermöglichen sollen und gleichzeitig eine Erwartungshaltung widerspiegelten. Dazu gehört das Konzept der Internalisierung externer Effekte, wie die Honorierung gesellschaftlicher Leistungen. Es sollen Strategien für das Zusammenführen der Akteure entwi- ckelt werden und mit den maßgeblichen Akteuren strategische Allianzen geschmiedet werden.

Freiherr von dem Bussche fasste die gwonnenen Eindrücke zum Arbeitsforum Landwirtschaft zusammen. Die Arbeitsgruppe des Rates sei derzeit auf einer wichtigen Stufe der Arbeit angelangt, auf der sie ihre grundsätzlichen Überlegungen nun in die Detailarbeit einfließen ließe. Sie werde die Anregungen des Forums in die zukünftigen Überlegungen aufnehmen. Die Erwartungen des Forums seien sehr unterschiedlich, sehr viele konstruktive Anregungen seien an den Rat gegeben worden. Der Rat sei guten Willens, diese zu berücksichtigen und in seine Arbeit aufzunehmen. In der Diskussion über die Erwartungen wäre vom Forum auch darauf hingewiesen worden, was der Rat nicht leisten könne.

Es zeige sich, dass sich eine sehr gute Gesprächskultur entwickelt. Voraussetzung für den Erfolg der Arbeit sei ein integrierendes und vernetztes Denken in einem anhaltenden Dialogprozess, an dem sich alle Akteure beteiligen. So könne der Rat seine Dialogfunktion erfüllen und auf eine effiziente Lösungsfindung hinwirken. Freiherr von dem Bussche dankte dem Forum für die rege Beteiligung und die Ermutigungen, die es dem Rat auf den Weg gibt.

\section{Nachhaltige Entwicklung vor dem Hinter- grund der Globalisierung (Prof. Dr. Klaus Töpfer)}

Die Abschlussrede der Auftaktveranstaltung des Rates für Nachhaltige Entwicklung hielt Prof. Dr. Klaus Töpfer, ehemaliger Bundesumwelt- und Wohnungsbauminister und derzeit Exekutivsekretär des Umweltprogramms der Vereinten Nationen (UNEP) sowie - last not least - Mitglied des Rates für Nachhaltige Entwicklung.

Töpfer ging zunächst auf die Darstellung der Zusammenhänge zwischen Umwelt und Entwicklung, der Ungleichgewichte zwischen Nord und Süd, ein. Hierzu zeigte er Weltkarten, die die Kontinente nicht in ihrer geografischen Ausbreitung darstellten, sondern in ihrer relative Größe entsprechend ihrer Wirtschaftskraft abbildeten. Diese Darstellungen machten das wirtschaftliche Übergewicht des "Nordens“ deutlich. Töpfer mahnte an, dass es nicht länger hingenommen werden dürfe, dass wir (die In- 
dustrieländer) preislos Humankapital (aus Entwicklungsländern) importierten und preislos dorthin Umweltbelastungen exportierten.

Töpfer begrüßte, dass die Europäische Union seit dem Gipfel des Europäischen Rats in Göteborg in diesem Jahr und durch den sogenannten Cardiff- und Lissabon-Prozess nun auch dem Leitbild der Nachhaltigkeit verpflichtet sei. Mit Sorge sei jedoch teilweise die Entwicklung in den Industriestaaten $\mathrm{zu}$ betrachten. Die Frage, inwieweit sich die Länder von der Nachhaltigkeit weg oder auf sie zu bewegen, wird zur Zeit - in Vorbereitung auf den Johannesburg-Gipfel - für die Weltregionen in assessment reports, für Europa durch die UN-ECE, zusammengestellt (die dann zu einem Bericht über die Lage der Welt verschmolzen werden sollen). Hier zeige sich: Obwohl Armut eine der Hauptursachen der Umweltzerstörung sei, geht der Trend weg von der Armutsbekämpfung, was man z. B. an den gesunkenen Ausgaben der Industrieländer für Entwicklungshilfe sehe (im Vergleich zu 1992 sind die Ausgaben 1999 um $18 \%$ gesunken). Töpfer verwies in diesem Zusammenhang auf die enorme Bedeutung der internationalen UNKonferenz zur Entwicklungsfinanzierung (FfD - Financing for Development) in Monterrey, Mexiko, im März 2002. UN, IWF, Weltbank und WTO diskutieren im Rahmen dieser Konferenz innovative Wege in der Entwicklungsfinanzierung, die den Anforderungen der Globalisierung gerecht werden sollen. Ein Erfolg in Johannisburg hänge in ganz entscheidendem Maße von einem Erfolg der FfD-Konferenz ab, so Töpfer. Die Entwicklungsländer bestünden zu Recht darauf, finanzielle Unterstützung bei einer Entwicklung in Richtung Nachhaltigkeit zu erhalten.

Töpfer ging auch auf das sog. „brain drain " ein: hochqualifizierte Menschen aus Entwicklungsländern wandern $a b$ in die reicheren Länder. Es sei für ihn erschreckend, dass dies z. B. in Deutschland nicht nur so hingenommen, sondern auch noch gefordert und gefördert werde (z. B., dass ,die Kinder schlauer Köpfe früher nachziehen dürfen“). Die Wirtschaft wolle kostenlos hochqualifizierte Menschen anwerben, für deren Ausbildung andere bezahlt haben, und somit ihr eigenes Versäumnis im Ausbildungsbereich ausgle ichen (der oben bereits angesprochene kostenlo- se Import von Humankapital). Den Entwicklungsländern gehen somit Arbeitskräfte verloren, die sie für den Auf- und Ausbau eigener Kapazitäten dringend benötigten.

Der Schutz vor Umweltzerstörung wiederum sei nur in reichen Ländern möglich: Je ärmer die Menschen sind, desto mehr Risiken sind sie ausgesetzt - je reicher, desto stärker können sie Vorsorge betreiben. Die Umweltund Gesundheits-Vorsorge sei aus Sicht der Entwicklungsländer oftmals ein Luxusgut.

Töpfer ging auch auf den Begriff der Nachhaltigen Entwicklung ein: Einerseits sei Nachhaltige Entwicklung eindeutig mehr als Umweltpolitik. Johannesburg dürfe auch kein Umweltgipfel, sondern müsse ein Nachhaltigkeitsgipfel werden. Die Entwicklungsfrage müsse stärker in den Mittelpunkt gerückt werden. Wenn aber auf der anderen Seite der Begriff ,nachhaltig" für ,,alles und jedes“ herangezogen werde, sei der Begriff nichts mehr wert: Er müsse also auch eingegrenzt werden.

Töpfer schloss seine Rede mit dem Hinweis auf einen neuen ,global deal“ ab: Entwicklungshilfe dürfe seitens der Geber nicht länger als Gnade betrachtet werden, sondern als unverzichtbare Zukunftsinvestition - ein global deal sei überlebensnotwendig für uns alle.

\section{Anmerkungen}

1) Kurzinformation zum Rat für Nachhaltige Entwicklung:

Der Rat für Nachhaltige Entwicklung wurde im April 2001 von Bundeskanzler Gerhard Schröder berufen. Er entwickelt Beiträge für eine nationale Nachhaltigkeitsstrategie der Bundesregierung, benennt konkrete Handlungsfelder und Projekte und soll die Bedeutung der Nachhaltigkeit als ein wichtiges öffentliches Anliegen unterstreichen. Auf der Rio-Nachfolgekonferenz in Johannisburg im Herbst 2002 werden alle teilnehmenden Länder ihre nationale Nachhaltigkeitsstrategie präsentieren. Dem Rat gehören 17 Personen des öffentlichen Lebens an: Dr. Volker Hauff (Vorsitzender), Philip Freiherr von dem Bussche, Jochen Flasbarth, Prof. Dr. Wolfgang Franz, Rainer Grohe, Roland Heinisch, Dr. Claus Hipp, Prof. Dr. Eberhard Jochem, Dr. Margot Käßmann, Prof. Dr. Edda Müller, Matthias Platzeck, Heinz Putzhammer, Prof. Dr. Josef Sayer, Prof. Dr. Klaus Töpfer, Prof. Dr. Fritz Vahrenholt, Hubert Weinzierl, Dr. 
Angelika Zahrnt

[Siehe auch die Darstellung des Rates in den TA-Datenbank-Nachrichten Nr. 2, Juni 2001, S. 72-74].

2) Der Rat für Nachhaltige Entwicklung stellt gegenwärtig seine Vorstellungen zu ausgewählten Zielen für Schwerpunktthemen der nachhaltigen Entwicklung im Internet vor und lädt zur Diskussion ein: http://www.nachhaltigkeitsrat.de/ dialog

\section{Kontakt}

Rat für Nachhaltige Entwicklung

Geschäftsstelle

Dr. Günther Bachmann

Reichpietschufer 50, 10785 Berlin

Tel.: +49 (0) 30 / 25491 - 780

Fax: +49 (0) $30 / 25491-785$

E-Mail: guenther.bachmann@ nachhaltigkeitsrat.de Internet: http://www.nachhaltigkeitsrat.de

》

\section{Vom Service zum Instrument - Auf der Suche nach einer Rolle für die Verkehrstelematik 8th World Congress on Intelligent Transport Systems}

Sydney, Australien, 30. September - 4. Oktober 2001

\section{Tagungsbericht von Günter Halbritter und Torsten Fleischer, ITAS}

Der 8. ITS Weltkongress, der nach den vorangegangenen Veranstaltungen in Berlin, Seoul, Toronto und Turin in diesem Jahr in Sydney stattfand, war wiederum eine bedeutende internationale Dialogplattform im Bereich der Anwendung neuer Informations- und Kommunikationstechniken (IuK-Techniken) im Verkehrswesen. Dieser Dialog zur Entwicklung und Einführung von Intelligent Transportation Systems (ITS), wie die Verkehrstelematik im englischsprachlichen Raum genannt wird, bzieht sich nicht nur auf die technischen Aspekte der neuen IuK-Techniken im Verkehrsbereich, sondern auch auf die organisatorischen, politischen, gesellschaftlichen und institutionellen Bedingungen der Technikeinführung. Diese, die ITS-Kongresse kennzeichnende, umfassende Betrachtungsweise der Technikeinführung machen diese Veranstaltungen auch für Technikfolgenabschätzungen sehr interessant. Den Rahmenbedingungen der ITS-Einführung wird in vielen Ländern, allen voran die USA, aber auch Australien, Japan und eine Reihe europäischer Länder, ein hoher Stellenwert eingeräumt. Dies zeigt sich nicht nur in der Schaffung gesetzlicher Grundlagen und in der Aufstellung von „nationalen“ Plänen zur Einführung von ITS, sondern auch darin, dass sich in vielen Staaten nationale - in der Regel aus Vertretern staatlicher Stellen und privatwirtschaftlicher Institutionen zusammengesetzte Organisationen im Sinne einer ,new governance" bei der Einführung dieser neuen Techniken engagieren.

Der diesjährige Kongress blieb selbstverständlich nicht unbeeinflusst von der Terrortragödie in New York und Washington. Dies drückte sich nicht nur dadurch aus, dass weit weniger amerikanische Teilnehmer als sonst üblich präsent waren. Auch wurde in den politischen Grundsatzerklärungen zu Beginn und am Ende der Tagung die Forderung erhoben, diese Ereignisse auch als neue Herausforderung an die zukünftige Gestaltung der Technik zu verstehen. Das Wegbleiben vieler US-amerikanischer Teilnehmer hatte zugleich zur Folge, dass die europäische Situation der Einführung von ITS deutlicher zur Geltung kam als dies bei früheren Veranstaltungen der Fall war.

Aus der Fülle der Veranstaltungen, die sich schwerpunktmäßig mit technischen Aspekten der Entwicklung und Einführung von IuK-Techniken im Verkehr befassten, waren aus der Perspektive der Technikfolgenabschätzung die politikorientierten Arbeitssitzungen von besonderem Interesse. Die technische Entwicklung im Bereich der Verkehrstelematik ist ohnehin nicht so sehr durch revolutionäre Veränderungen als vielmehr durch eine evolutionäre Entwicklung gekennzeichnet. Dies zeigten nicht nur die Vortrags- und Diskussionsveranstaltungen, sondern auch die begle itende Ausstellung. Dort wurde viel Bekanntes in überarbeiteter und perfektionierter Form zur Schau gestellt, innovative neue Ansätze zur Lösung von Verkehrsproblemen durch verstärkte Nutzung von IuK-Techniken waren kaum zu finden. 